\title{
Cistitis papilar y polipoide que simula un carcinoma urotelial papilar
}

\author{
Polypoid and papillary cystitis simulating papillary \\ urothelial carcinoma
}

Jesús Sebastián Muruato-Araiza, ${ }^{1}$ Alan Martínez-Salas, ${ }^{1}$ Irving Reyna-Blanco, ${ }^{1}$ Aldo Jiménez-García, ${ }^{1}$ Mauricio Cantellano-Orozco, ${ }^{1}$ Jesús Cienfuegos-Meza, ${ }^{2}$ Sara Paraguirre-Martínez, ${ }^{2}$ Carlos Martínez-Arroyo, ${ }^{1}$ Jorge G. Morales-Montor, ${ }^{1}$ Gerardo Fernández-Noyola, ${ }^{1}$ Carlos Pacheco-Gahbler ${ }^{1}$

\section{Resumen}

ANTECEDENTES: La cistitis polipoide es una alteración provocada por la irritación de la mucosa vesical debida al uso crónico de sondas transuretrales.

CASO CLínICO: Paciente masculino de 74 años, con antecedente de diabetes tipo 2, infarto agudo de miocardio (tres ocasiones) y colocación de tres stents; tabaquismo intenso ( 35 paquetes-año) y hematuria macroscópica, sin formación de coágulos, de un año de evolución, de curación espontánea. Un mes previo a su ingreso a la unidad médica, la urotomografía evidenció un defecto de llenado vesical en fase de eliminación. Durante su evaluación el examen general de orina reportó microhematuria; el resto de los estudios de laboratorio no mostró alteraciones. En la urotomografía nuevamente se observó un defecto de llenado vesical en fase de eliminación, de 2.3 $\times 1.5 \mathrm{~cm}$. En la cistoscopia se identificó una tumoración sésil y papilar, dependiente de la pared lateral derecha, por lo que se efectuó la resección transuretral. El estudio histopatológico reportó cistitis papilar-polipoide. La sonda transuretral se retiró después de tres días posquirúrgicos. Hoy día se mantiene en seguimiento (cinco meses), sin evidencia de recurrencia tumoral.

CONCLUSIÓN: La cistitis polopide-paliar es una alteración que debe considerarse en el diagnóstico diferencial del carcinoma urotelial de vejiga.

PALABRAS CLAVE: Cistitis polipoide; cistitis papilar; carcinoma urotetial.

\section{Abstract}

BACKGROUND: Polypoid cystitis is an alteration caused by irritation of the bladder mucosa due to the chronic use of transurethral catheters.

CLINICAL CASE: A 74-year-old man had a history of type 2 diabetes mellitus, acute myocardial infarction (on three occasions), and intense smoking (35 packs/year). He also presented with gross hematuria with no clot formation of one-year progression that resolved spontaneously. One month prior to admission to our medical unit, computed tomography urogram revealed a bladder filling defect in the elimination phase. The patient's evaluation revealed microhematuria in the urinalysis and there were no alterations in the rest of the laboratory studies. A urogram once again identified a bladder filling defect in the elimination phase, measuring $2.3 \times 1.5 \mathrm{~cm}$. Cystoscopy showed a sessile, papillary tumor dependent on the right lateral wall, thus transurethral resection was performed. The histopathologic study stated polypoid and papillary cystitis. The transurethral catheter was removed on postoperative day three. The patient remains in follow-up (five months), with no signs of tumor recurrence.

CONCLUSION: Polypoid and papillary cystitis is an alteration that should be considered in the differential diagnosis of urothelial carcinoma of the bladder.

KEYWORDS: Polypoid cystitis; Papillary cystitis; Urothelial carcinoma.
${ }^{1}$ División de Urología.

2División de Anatomía Patológica.

Hospital General Dr. Manuel Gea González, SSA, Ciudad de México.

Recibido: octubre 2017

Aceptado: agosto 2018

Correspondencia

Jesús Sebastián Muruato Araiza smaraiza@hotmail.com

Este artículo debe citarse como Muruato-Araiza JS, Martínez-Salas A, Reyna-Blanco I, Jiménez-García A, Cantellano-Orozco M, ParaguirreMartinez S, Martínez-Arroyo C, Morales-Montor JG, Fernández-Noyola G, Pacheco-Gahbler C. Cistitis papilar y polipoide que simula un carcinoma urotelial papilar. Rev Mex Urol. 2018 sept-oct;78(5):375-378.

DOI: https://doi.org/10.24245/revmexurol.v78i5.1748 


\section{ANTECEDENTES}

La cistitis polipoide de fase crónica (cistitis papilar) es una alteración provocada por la irritación de la mucosa vesical, similar a ciertos tipos de lesiones neoplásicas, como el carcinoma urotelial. La cistitis papilar suele afectar a pacientes con cateterismo vesical permanente o intermitente, litiasis vesical, fístula colovesical y crecimiento prostático obstructivo.

A continuación se reporta el caso de un paciente con cistitis polipoide, con antecedente de tabaquismo intenso y hematuria macroscópica como síntoma inicial.

\section{CASO CLÍNICO}

Paciente masculino de 74 años, con antecedente de diabetes mellitus tipo 2, infarto agudo de miocardio (tres ocasiones) y colocación de tres stents en una institución médica ajena a la actual; tabaquismo intenso (35 paquetes-año) y hematuria macroscópica sin formación de coágulos de un año de evolución, de curación espontánea. Un mes previo a su ingreso a nuestra unidad, la urotomografía evidenció defecto de Ilenado vesical en fase de eliminación. Durante su evaluación, el examen general de orina reportó microhematuria; el resto de los estudios de laboratorio no mostró alteraciones.

La urotomografía (Figura 1) efectuada en nuestro hospital evidenció, nuevamente, defecto de llenado vesical en fase de eliminación, de $2.3 \times 1.5 \mathrm{~cm}$. La cistoscopia diagnóstica con anestesia general, balanceada con cistoscopio Wolf $19 \mathrm{Fr}$, identificó una tumoración sésil y papilar, dependiente de la pared derecha (Figura 2), por lo que se efectuó resección transuretral. Se obtuvo una biopsia con corte frío de la base del tumor. El estudio histopatológico del tejido de fronda tumoral, base y lecho reportó cistitis papilar-polipoide, músculo detrusor sin altera-
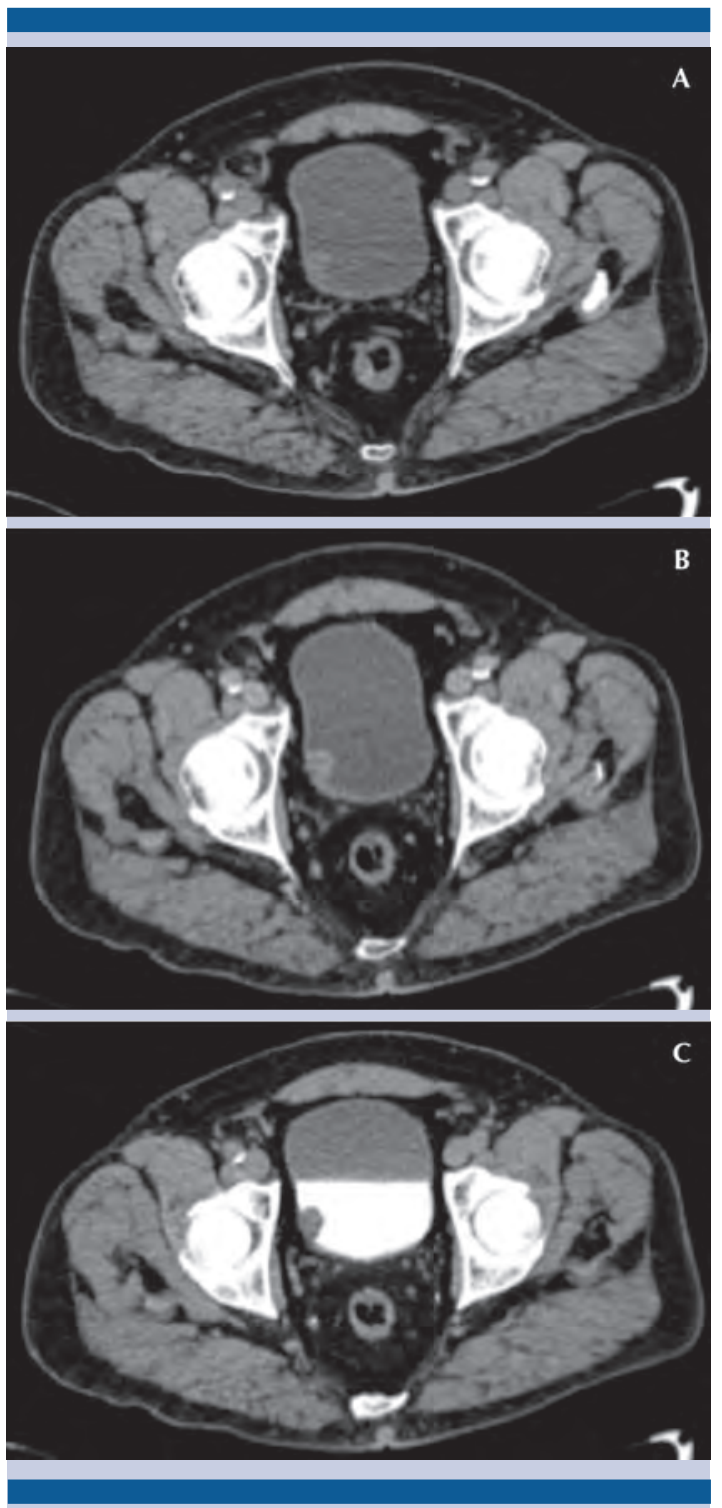

Figura 1. Urotomografía en fase simple con imagen hiperdensa dependiente de la pared lateral derecha de $31 \mathrm{UH}$ (A), fase arterial con reforzamiento de 43 UH (B) y fase de eliminación que evidencia el defecto de llenado vesical, dependiente de la pared derecha de $2.3 \times 1.5 \mathrm{~cm}$.

ciones y tejido de lecho tumoral escaso, además de músculo con evidencia de daño térmico. La sonda transuretral se retiró después de tres días posquirúrgicos. Actualmente, el paciente 


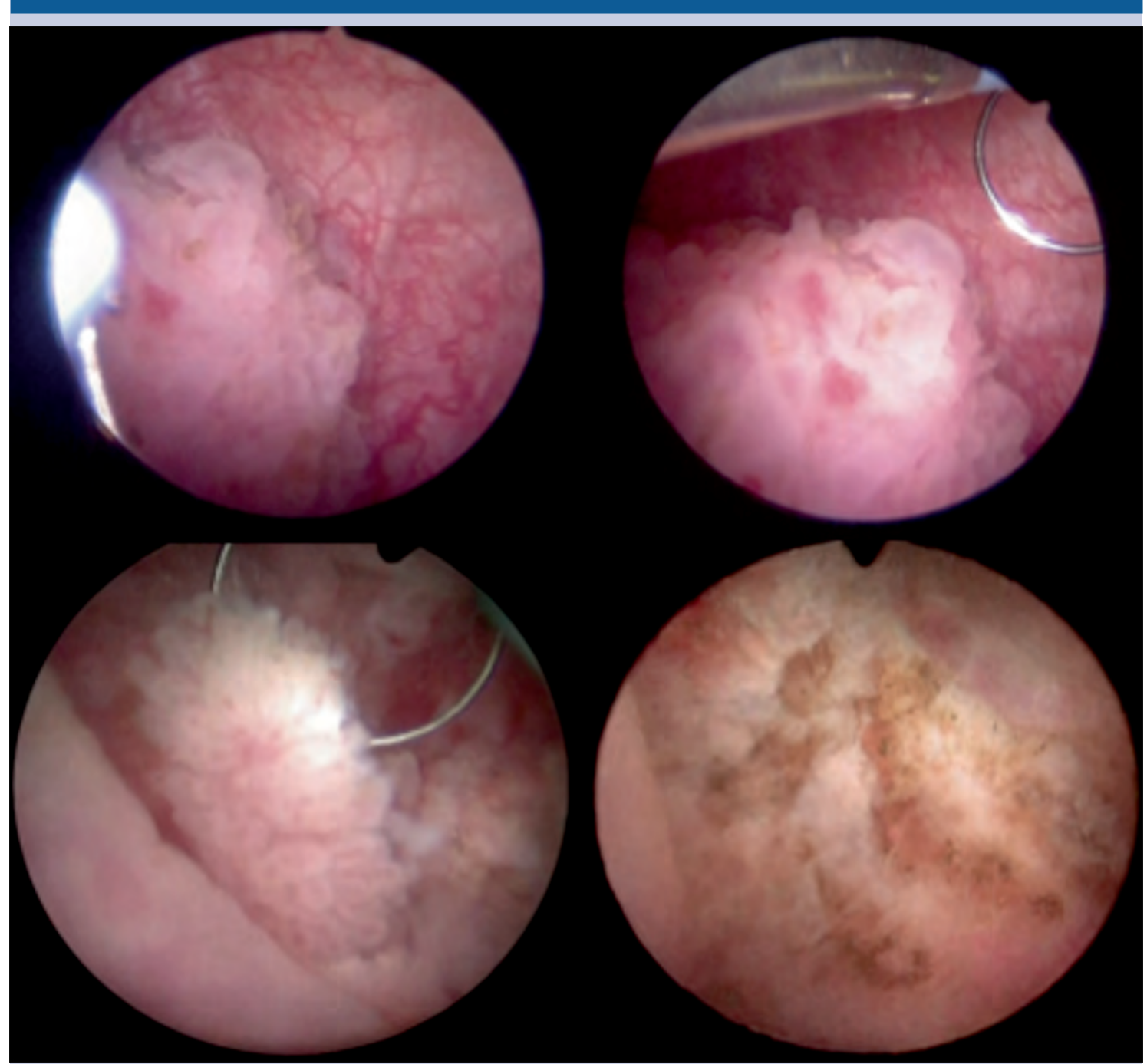

Figura 2. Cistoscopia diagnóstica y resección transuretral. Se observa una tumoración sésil papilar, dependiente de la pared derecha.

permanece en seguimiento (cinco meses), sin evidencia de recurrencia tumoral.

\section{DISCUSIÓN}

La cistitis polipoide, descrita inicialmente por Mostofi' es una alteración benigna ocasionada por una lesión en la mucosa vesical, principalmente por el uso crónico de sondas transuretrales. ${ }^{2}$ También se ha observado en pacientes con litiasis vesical y crecimiento prostático obstructivo; ${ }^{3}$ sin embargo, nuestro caso no tenía antecedentes de lo expuesto en la bibliografía, lo que dificultó establecer el diagnóstico.

Las manifestaciones clínicas incluyen síntomas de vaciamiento vesical y hematuria macroscópica; no obstante, la mayoría de los casos no manifiesta lesiones. ${ }^{4}$ 
La cistitis polipoide puede confundirse con alguna neoplasia urotelial papilar; por lo tanto, se requiere efectuar el estudio histopatológico para establecer el diagnóstico preciso. Los hallazgos más sobresalientes incluyen: fondo edematoso con grosor urotelial relativamente normal y lesiones menores de $5 \mathrm{~cm}$.

El ensayo de Epstein y su grupo, efectuado en el Hospital Johns Hopkins, reportó 41 de 155 pacientes mal diagnosticados con neoplasias uroteliales papilares, que incluían papiloma o carcinoma papilar de alto y bajo grados, este último con mayor prevalencia. ${ }^{2}$ Una de las principales características que genera confusión es que mientras más pasa el tiempo, la fronda de la cistitis polipoide provoca menor edema y se remplaza por tejido fibroso, proceso conocido como "cistitis papilar" (una lesión similar a las neoplasias papilares uroteliales), que afecta a $39 \%$ de los casos.

\section{CONCLUSIÓN}

La cistitis polipoide y papilar es una alteración que debe considerarse en el diagnóstico diferen- cial del carcinoma urotelial de vejiga, incluida la obtención de una biopsia. En estos casos se requiere la descripción correcta de las lesiones y en pacientes sin antecedentes de tumoración vesical es necesaria la resección quirúrgica y descartar la coexistencia de cáncer vesical.

\section{REFERENCIAS}

1. Mostofi FK. Potentialities of bladder epithelium. J Urol. 1954;71:705-714.

2. Lane Z, Epstein JI. Polypoid-papillary cystitis: a series of 41 cases misdiagnosed as papillary urothelial neoplasia. Am J Surg Pathol 2008;32:758. DOI: 10.1097/PAS.0b013e31816092b5

3. Kilic S, et al. Polypoid cystitis unrelated to indwelling catheters: a report of eight patients. Int Urol Nephrol 2002;34:293-297.

4. Ozaki $\mathrm{K}$, et al A case of polypoid and papillary cystitis mimicking an advanced bladder carcinoma with invasion of perivesical fat. Urol Ann 2014;6:72-4. DOI: 10.4103/09747796.127016

5. Ekelund P, Johansson S. Polypoid cystitis: a catheter associated lesion of the human bladder. Acta Pathol Microbiol Scand [A]. 1979;87A:179-184.

6. Kim SH, Yang DM, Kim NR. Polypoid and papillary cystitis mimicking a large transitional carcinoma in a patient without a history of catheterization: Computed tomography and magnetic resonance findings. J Comput Assist Tomogr $2004 ; 28: 485-7$ 